\title{
A STRATEGIC APPLICATION OF FAST FOURIER TRANSFORM AS A NOVEL TOOL TO EVALUATE THE EXTENT OF NEURONAL INSULT IN RAT MODEL OF FOCAL CEREBRAL ISCHEMIA
}

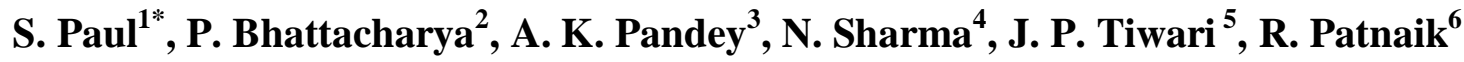 \\ 1, 2,3,4,6 School of Biomedical Engineering, Institute of Technology \\ ${ }^{5}$ Department of Electrical Engineering, Institute of Technology \\ Banaras Hindu University, Varanasi-221005, India \\ *e-mail: sudip.paul.bhu@ gmail.com (Corresponding author)
}

\begin{abstract}
The present work envisages mathematical modeling of induced focal cerebral ischemia in animal model using EEG data with the help of Fast Fourier Transformation method. Amongst several analysis methods, spectral analysis methods are important because it detects the frequencies and characteristics changes of brain waveforms depending on the brain function affected from disorders and physiological state. There are many applications of FFT, and the most important being that it is one of the basic conventional spectral analysis methods. However, it has some limitations, for instance, it adds contributions in the low frequency region which are not present in the original signal, and necessitates the use of windowing for decreasing the error rate. The present analysis was undertaken to ensure actual correlation of the different mathematical paradigms. EEG data were obtained from different regions of rat brain and were processed by FFT modeling in MATLAB platform. The assessment of long lasting functional outcome and to prevalent classical approach to study stroke was necessitated and therefore highly recommended to evaluate the efficacy of therapeutic strategies in relation to EEG in animal model of brain stroke. This mathematical modeling specifically Power Spectrum Density analysis was done to correlate the different prevalent condition of rat brain function.
\end{abstract}

Keywords: EEG, Mathematical modeling, Focal Cerebral Ischemia, Piroxicam, Fast Fourier Transformation, Power Spectrum Density

\section{INTRODUCTION}

The pathology associated with the majority of ischemic stroke in animal is an inflammatory response [1]. Inflammation is a normal, protective response to tissue injury caused by physical trauma, noxious chemicals or microbiological agents. Experimental studies have reported the role of inflammation in ischemic insult [2] and it is largely accepted that ischemic stroke is characterized by activation of multiple cellular cascades and can lead to extensive cellular insult [3] due to several pathogenic factors acting in a coordinated fashion [4].

Focal cerebral ischemia is a multi-faceted condition which involves a myriad of potent death mediators having a contribution to the overall cellular insult. Ischemic stroke is one of the most common neurological disease which ranks third in the leading cause of death after heart disease and cancer. The poor prognosis of cerebral ischemia is contributed mainly to the acute irreversible loss of brain cells before the patient receives medical aid, which is exacerbated by the void in delayed clinical intervention, options to protect against secondary insult [4]. Ischemic stroke is most often caused by the occlusion of cerebral artery and these results into a detrimental pathological cascade of cellular events which includes loss of cerebral blood flow, breakdown of Blood Brain Barrier and oedema [4]. As a result of disrupted blood flow, there is an oxygen deficit which brings about metabolic changes 
in the ischemic area $[5,6]$. In the minutes and hours of cerebral ischemia, there is disruption of ionic homeostasis, release of intracellular potassium, accumulation of intracellular calcium and sodium, loss of membrane potential, depolarization of neurons and glia [7, 8], increase in mitochondrial NADH level, depletion in ATP synthesis [9], increase in the level of extracellular glutamate and production of cytotoxic free radicals, which leads to further cellular injury, inflammation, necrosis and apoptosis in the area adjacent to the initial insult [10] causing long term pathogenic effect. The mitochondrial injury due to the reduced oxygen supply during cerebral ischemia is gradual and spreads from acute damage in the core area towards moderate damage in the peripheral regions known as the penumbra $[6,9,11]$. The present work envisages creation of a rat model of focal cerebral ischemia, its validation and to explore mathematic modelling as a tool to evaluate degree of neuronal insult due to such injury. Our objective is to design a methodology to dissect the consequences of ischemia and to pre-empt the pathology with our proposed mathematical model.

\section{MATERIALS AND METHODS}

We have tried to create a rat model of ischemia / reperfusion which can mimic actual pathology of human stroke. We have validated our model through biochemical studies. Further we conducted EEG studies in three rat groups i.e. Control, Stroke and Drug administered and tried to co-relate different brain signals obtained with our mathematical tool on MATLAB platform. The drug was used to minimise the effect of ischemia. Further we have formulated an algorithm which can provide information regarding the pathological state and even pre-empt the pathological condition by analysing different brain signals at different pathology. A description of the animals and drugs used, methods for induction of focal ischemia, evaluation methods based on motor function testing and the present signal processing methods are presented below.

\subsection{Animals}

Male Charles Foster rats (6 weeks, $230 \pm 10$ g) in-bred at the Central Animal House of Banaras Hindu University (Registration No.- 542/AB/CPCSEA) were used for the experiments. Animals were kept under standard laboratory conditions maintained with the highest standards of animal care and housing. Animals were provided with a medicated commercial diet having standard ingredients. They were allowed free access to food and water and maintained at $12 \mathrm{~h}$ day/night cycle. The approved standard procedures and the institutional animal ethical committee guidelines were followed throughout the experiments.

\subsection{Drugs}

As per previous studies Non-steroidal anti-inflammatory drugs (NSAIDs) have been proved to have neuroprotective potentials [12]. Piroxicam, a NSAID, rescue neurons from hypoxia / reoxygenation by COX-2 inhibition [13] at cellular level (in vitro). We have tried to justify this in rat model (in vivo) for the very first time. Piroxicam and most of the other chemicals were purchased from Sigma (St Louis, MO, USA).

\subsection{Induction of Focal cerebral ischemia}

Focal cerebral ischemia was induced by middle cerebral artery occlusion (MCA) with modified intraluminal technique [14]. Rats were anesthetized by the administration of a combination of ketamine (75 mg / kg b.w.) and xylazine (10 mg / kg b.w.) and then transferred to the surgical table with a heating pad to maintain a constant body temperature of $37 \pm 0.5^{\circ} \mathrm{C}$. Rat was placed in a supine position with forelimbs fixed on the table by tape and the fur on the ventral neck was shaved and the 
skin was cleaned by $0.5 \%$ Betadine and $75 \%$ alcohol. Midline incision in the neck was done to expose the left common carotid artery (CCA). Muscle fascia was separated further to expose external carotid artery (ECA) and internal carotid artery (ICA). A $5.0 \mathrm{~cm}$ length 3-0 monofilament nylon suture (Ethicon) was introduced into the CA lumen through a small nick and gently pushed from ICA lumen to block the origin point of MCA. Approximately 18-22 mm length of nylon filament was inserted to reach the MCA blockade site from the bifurcation point. The ECA stump was clamped around the intraluminal nylon suture to prevent bleeding. Reperfusion was done by gently removing the filament after $1 \mathrm{hr}$ of ischemia. Animals were allowed to recover from anesthesia and on regaining the righting reflex, were transferred to polypropylene cages in the animal room with temperature maintained at 26 $\pm 2.5^{\circ} \mathrm{c}$ with food and water supply in ad-libitum.

In control-operated animals, all the procedures were carried on except the insertion of nylon filament. For administration of the drug, we found that 30 min i.p pretreatment of Piroxicam renders maximum neuroprotection as revealed from staining and scoring (data not shown). Hence Piroxicam was administered i.p 30 min pre in the stroke group.

\subsection{Motor function test}

Motor function test was performed $12 \mathrm{~h}$ after MCA occlusion using Rota rod tests. The rats were first conditioned on the Rota rod (IITC, USA) at a constant speed of $10 \mathrm{rpm}$ until they achieved the criterion of remaining on the rotating spindle for $60 \mathrm{~s}$. Thereafter, each rat received a single trial on the accelerating Rota rod in which the speed of the spindle was increased from 5 to $20 \mathrm{rpm}$ over a period of $180 \mathrm{sec}$, which was taken as the baseline value. The time for which the rats remained in the accelerating Rota rod was again tested at $12 \mathrm{~h}$ of MCA occlusion.

\subsection{Physiological evaluation}

Prior to MCAo surgery, rectal temperatures and neurologic function were assessed and baseline EEG recordings were made continuously for at least 30 mins. Animals were then removed from their custom recording chambers for MCAo surgery [15]. Immediately after surgery, animals were returned to recording chambers and continuous EEG recordings were again initiated. For the following $2 \mathrm{~h}$ post-MCAo, continuous digital and polygraph EEG records were obtained. By performing this we have tried to establish an experimental exclusion criterion and to determine the effect of Piroxicam in optimized dose to improve cortical EEG activity in injured rats. Changes in EEG amplitude were quantified with the use of Power spectral analysis and data reported as percent EEG recovery compared.

\subsection{Signal Processing}

The most widespread signal processing method applied to signals is the spectral analysis which was used to analyze the EEG signals for the different conditions of the animal brains. For this, EEG signals in the time domain were sampled at an appropriate frequency. Sampled signals were grouped as frames that contain evident sample numbers. The power spectral density $\mathrm{p}(\mathrm{f})$ was found for each window by applying conventional and modern spectral analysis method such as Fast Fourier Transform (FFT) [16]. This produced frequency spectrum graphs of the EEG signals. The best appropriate length of the used frame depends on the steadiness of signal and sampling frequency. In this study, the sampling rate was 256 , because sampling frequency is low and the numbers of samples are few as well.

\subsection{Algorithm}

To analyse the EEG signal, the basic chain of operations consist of 
- Acquisition of data and remove artifacts from the data: for this case the suitable filters were programmed and applied for the signals.

- Processing: this methodology was done in MATLAB platform with suitable commands.

- Numerical analysis: results were taken in .txt format and graphs were taken into consideration.

The algorithms are shown in Fig.1.

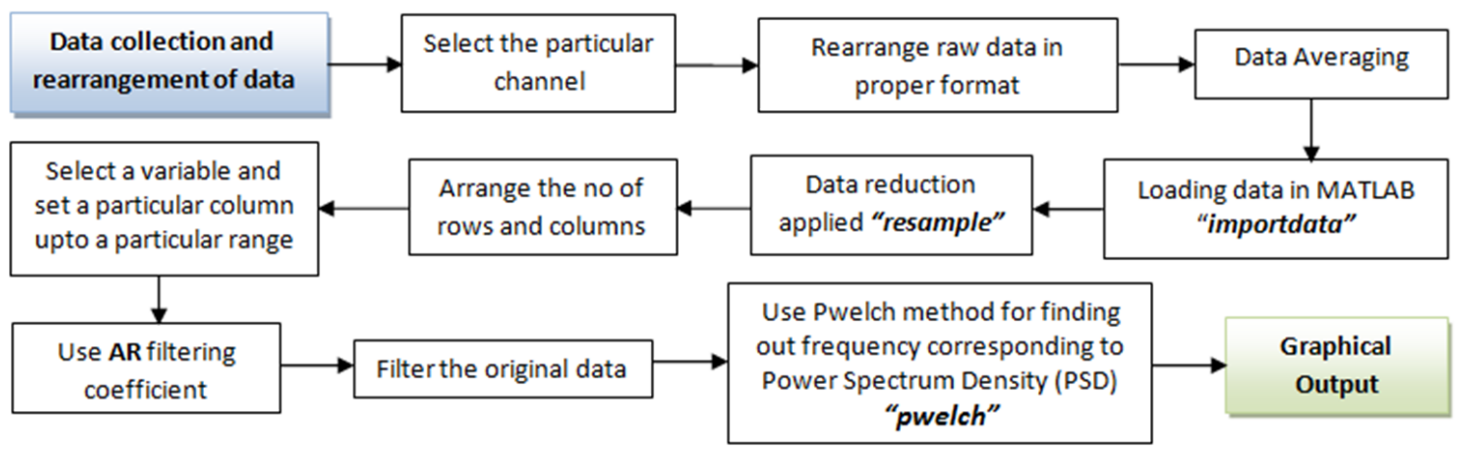

Fig.1: Block diagram representation of Algorithm

\section{RESULTS}

The total 42 rats were used in three different groups where $n=14$ in each group. Piroxicam was

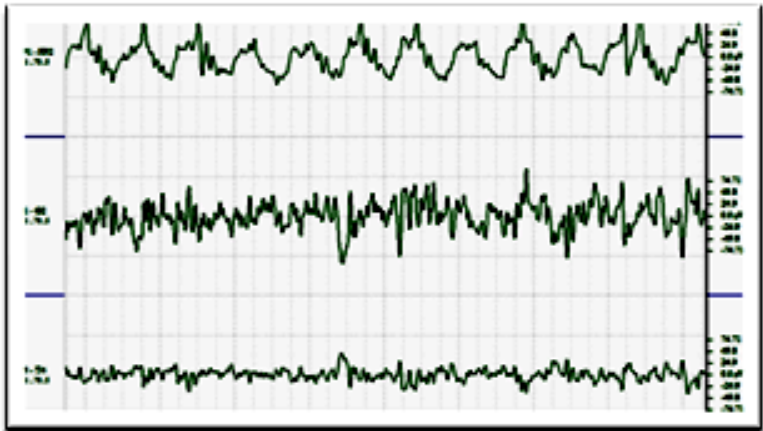

Fig.2: Normal rat brain EEG signal

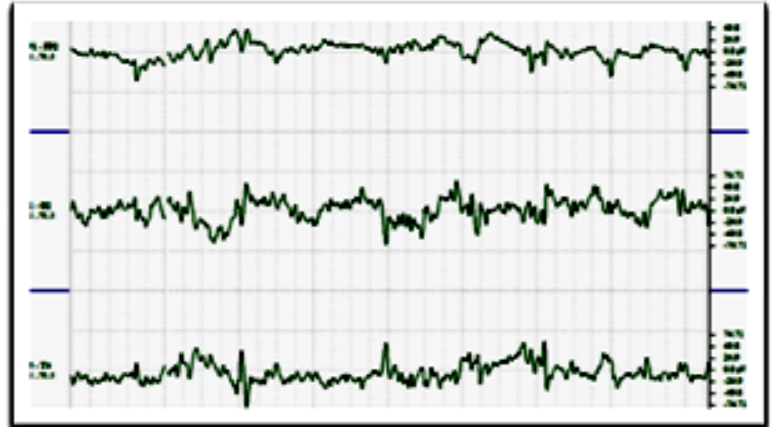

Fig.3: Stroke rat brain EEG signal

administered i.p 30 min pre-induction of focal cerebral ischemia as correlated with the staining and biochemical studies (data not shown). Experiments were carried out with these three sets and the results are represented as mean \pm SD. Statistical significant was analyzed using ANOVA followed by Turkey's post hoc test. Differences of $\mathrm{P}<0.05$ were considered significant. The Experiments were done in same time of a day and the signals which were recorded by the polygraph instrument are

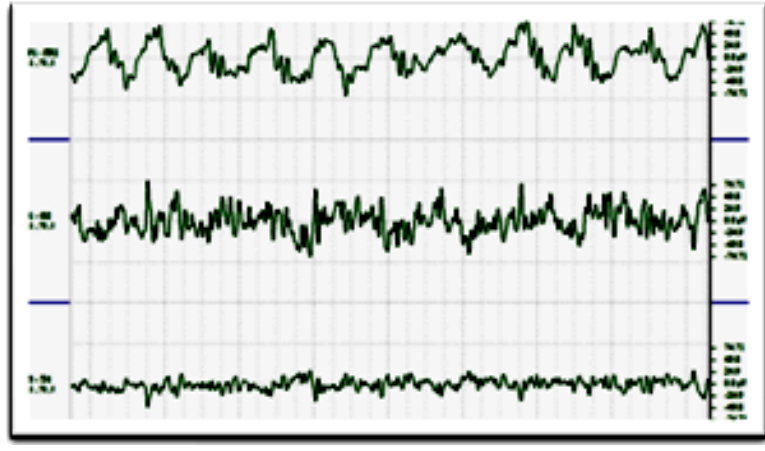

Fig.4: Stroke + drugged rat brain EEG signal shown in Figs. 2, 3 and 4. 
In this study we tried to analyze and recognize the alterations of EEG signals in three groups of rats, i.e. normal, stroke and drug administered. EEG analysis was done on the basis of mathematical modeling through Power Spectrum Density (PSD). The changes in PSD in the three different animal groups at different physical locations of the brain (Frontoparietal, Occipital and Temporal) are shown in Figs. 5, 6 and 7 respectively at different frequency bands of EEG (Delta, Theta, Alpha and Beta). The percentages of recovery, with reference to control, due to administration of Piroxicam are shown in Fig. 8.
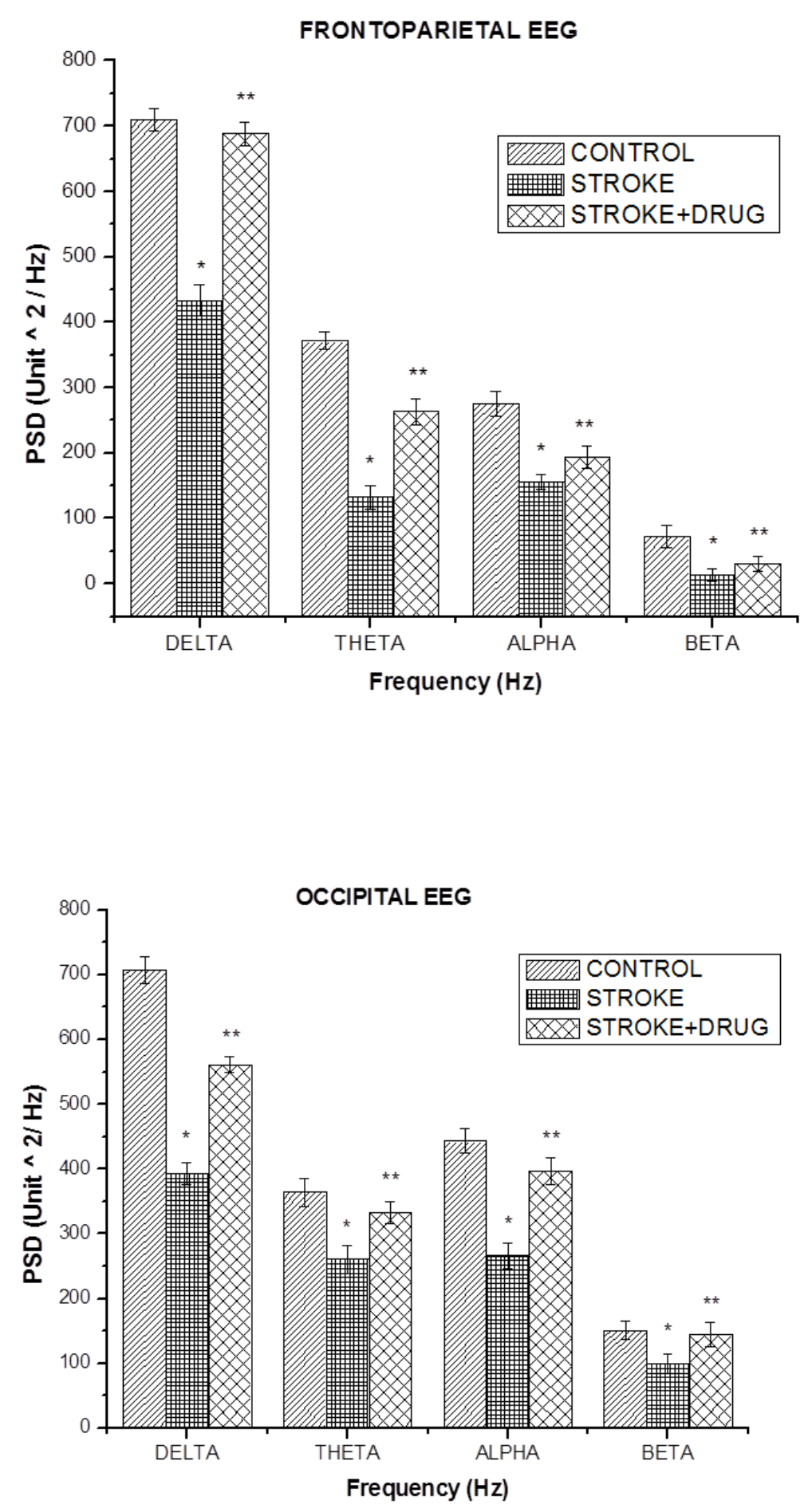

Fig. 5: Changes in EEG signal in the frontal region of rat brain expressed as mean $\pm \mathrm{SD}(* \mathrm{vs}$ control and **vs stroke)
Fig. 6: Changes in EEG signal in the occipital region of rat brain expressed as mean $\pm \mathrm{SD}$ (*vs control and **vs stroke) 


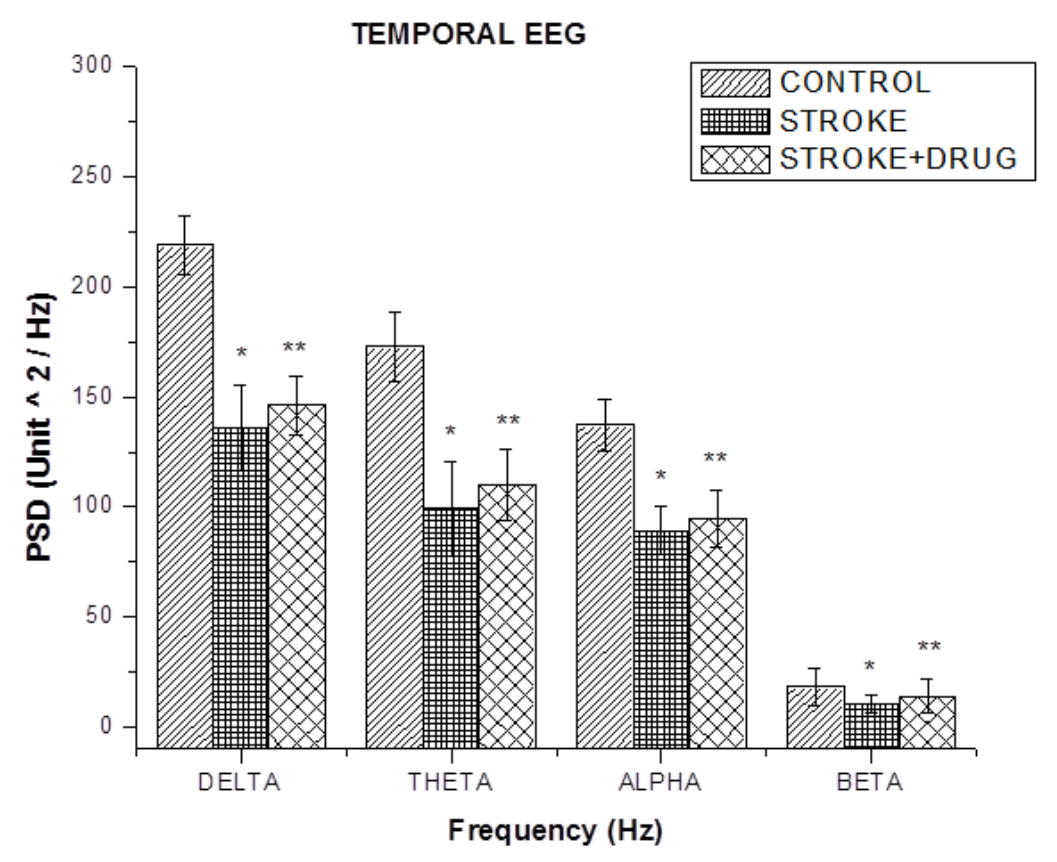

Fig. 7. Changes in EEG signal in the temporal region of rat brain expressed as mean $\pm \mathrm{SD}(* \mathrm{vs}$ control and **vs stroke)

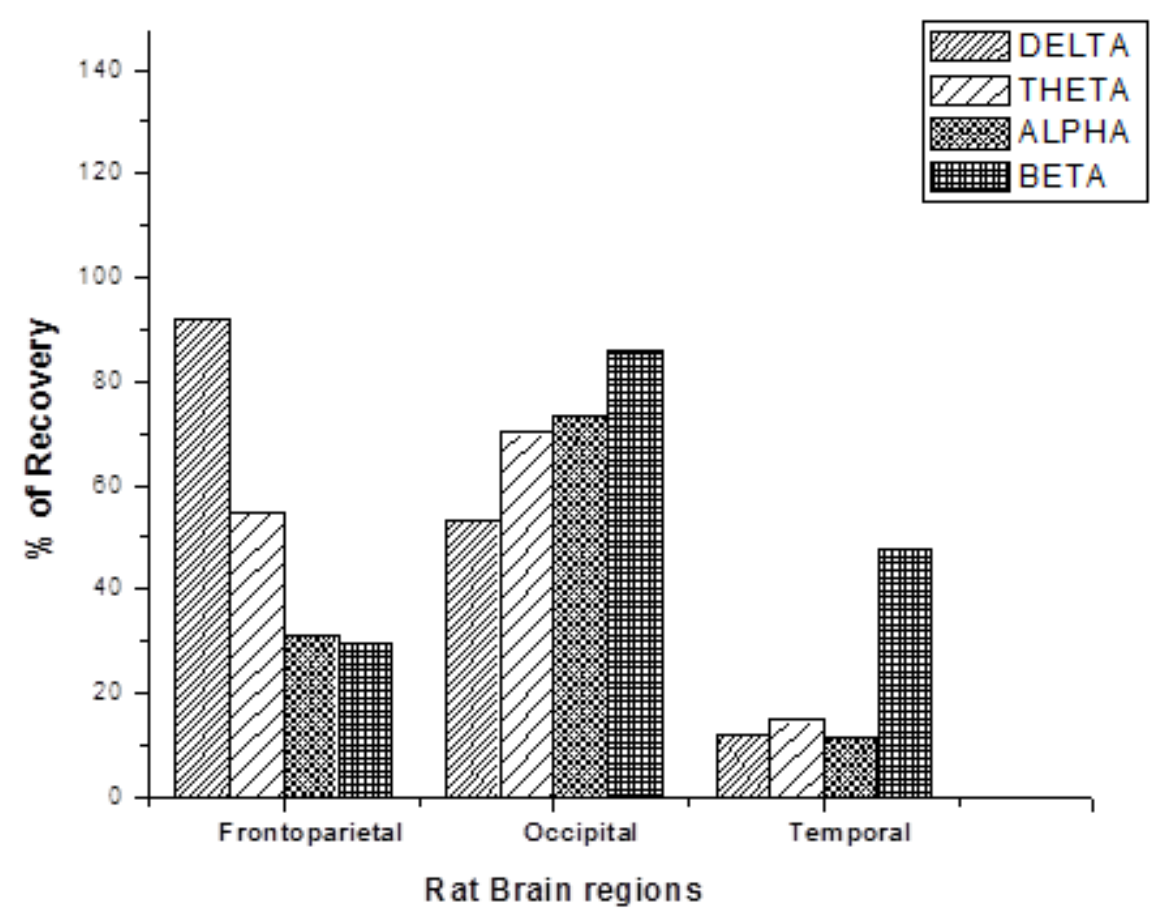

Fig. 8: Changes in different frequency bands in different rat brain region i.e. the percentage of recovery after administration of drug (with reference to Control)

\section{DISCUSSION}

Past studies have evolved the notion that following ischemia reperfusion injury, there arises abnormal cortical electrical discharge which is a result of altered neuronal firing. These abnormal EEG discharge analysis measures have been traditionally used to detect residual neural dysfunctions after cerebral ischemia. It is feasible to think that advanced EEG research and its intervention can provide more clear information regarding the long lasting neural impairment in subjects suffering from cerebral ischemia. Here we have tried to put forward an observation with validation to verify that 
EEG abnormalities may be present in ischemia-reperfusion model in addition to other signs and symptoms. Specifically, our current findings have clearly documented that EEG have significantly changed in several brain regions following cerebral ischemia in animal model. In this study it was tried to analyze and identify the alterations of EEG signals in three groups of rat, i.e. normal, stroke and drug administered. EEG analysis was done on the basis of mathematical modeling with the help of Fast Fourier Transformation and Power Spectrum Density (PSD) analysis. It was observed that there are significance changes (Figs. 5, 6 and 7) in the PSD at frequency band domains. Piroxicam administered rats showed a significant recovery towards achieving normal rhythm (Fig. 8). However, the recovery was not the same in all the brain locations and in all the EEG frequency bands. Particularly this was low for the Delta, Theta and Alpha bands in the Temporal region. This finding may lead to new research into the effects of ischemic stroke on the brain.

\section{CONCLUSIONS}

The work presented shows the value of EEG in distinguishing normal and ischemic stroke conditions. Recognizing such a pattern may serve as a diagnostic tool to understand the degree of neuronal insult in the specific region of brain following brain stroke. So, the mathematical modelling used can serve as a tool to recognise altered brain pathology at an early stage, and used in conjunction to other conventional diagnostic methods, may be able to diagnose patients predisposed to brain stroke.

\section{REFERENCES}

1. Ross. R., "Atherosclerosis - an inflammatory disease". Review New Engl. J. Med, vol. 340(2), pp. 115-126, 1999.

2. Becker, K., Kindrick, D., Relton, J., Harlan, J., and Winn, R., "Antibody to the alpha4 integrin decreases infarct size in transient focal cerebral ischemia in rats". Stroke. vol. 32, 206-211, 2001.

3. Sweeney, M. I., Yager, J. Y., Walz, W. and Juurlink, B.H., "Cellular mechanisms involved in braim ischemia". Can. J. Physiol. Pharmacol. vol. 73, pp. 1525-1535, 1995.

4. Dirnagl, U., Iadecola, C., and Moskowitz, M.A. "Pathobiology of ischemic stroke: An integrated view". Trends Neurosci. vol. 22, pp. 391-397, 1999.

5. Siesjo, B.K. "Acidosis and ischemic brain damage". Neurochem. Pathol. vol. 9. pp. 31-88, 1988.

6. Lipton, P., "Ischemic cell death in brain neurons". Physiol Rev. vol. 79. pp. 1431-1568, 1999.

7. Katsura, K., Kristian, T. And Siesjo, B.K. Biochem. Soc. Trans. vol. 22. pp. 991-996, 1994.

8. Martin, R.L., Lloyd, H.G. and Cowan, A.I., Trends Neurosci. vol. 17. pp. 251-257, 1994.

9. Sims, N.R., and Anderson, M.F. "Mitochondrial contributions to tissue damage in stroke". Neurochem. Int. vol. 40. pp. 511-526, 2002.

10. Budd, S.L., "Mechanisms of neuronal damage in brain hypoxia / ischemia: focus on the role of mitochondrial calcium accumulation". Pharmacol. Ther. vol. 80. pp. 203-229, 1998.

11. Astrup, J., Siesjo, B.K. and Symon, L. Stroke. vol. 12. pp. 723-725, 1981.

12. Mishra, V., Verma, R. and Raghubir, R., "Neuroprotective effect of flurbiprofen in focal cerebral ischemia: the possible role of ASIC1a", Neuropharmacology, vol. 59, pp 582-588, 2010.

13. Vartiainen N, Huang CY, Salminen A, Goldsteins G, Chan PH and Koistinaho J., "Piroxicam and NS-398 rescue neurones from hypoxia/reoxygenation damage by a mechanism independent of cyclo-oxygenase inhibition", J Neurochem, vol.76(2), pp 480-9, 2001.

14. Longa, E. Z., Weinstein, P. R., Carlson, S., and Cummins, R., "Reversible middle cerebral artery occlusion without craniectomy in rats", Stroke. vol. 20. pp. 84-91, 1989. 
15. Jed A. Hartings, Anthony J. Williams, and Frank C. Tortella., "Occurrence of nonconvulsive seizures, periodic epileptiform discharges, and intermittent rhythmic delta activity in rat focal ischemia". Experimental Neurology. vol. 179. pp. 139-149, 2003.

16. Akin, M., and Kemal Kiymik, M., "Application of Periodogram and AR Spectral Analysis to EEG signals", Journal of Medical Systems. vol. 24. pp. 247-256, 2000. 